\title{
Prediction Based on Generalized Order Statistics from a Mixture of Rayleigh Distributions Using MCMC Algorithm
}

\author{
Tahani A. Abushal ${ }^{1}$, Areej M. Al-Zaydi ${ }^{2}$ \\ ${ }^{1}$ Department of Mathematics, Umm Al-Qura University, Makkah Al-Mukarramah, KSA \\ ${ }^{2}$ Department of Mathematics, Taif University, Taif, KSA \\ Email: tabushal@yahoo.com, aree.m.z@hotmail.com
}

Received May 15, 2012; revised June 16, 2012; accepted June 30, 2012

\begin{abstract}
This article considers the problem in obtaining the maximum likelihood prediction (point and interval) and Bayesian prediction (point and interval) for a future observation from mixture of two Rayleigh (MTR) distributions based on generalized order statistics (GOS). We consider one-sample and two-sample prediction schemes using the Markov chain Monte Carlo (MCMC) algorithm. The conjugate prior is used to carry out the Bayesian analysis. The results are specialized to upper record values. Numerical example is presented in the methods proposed in this paper.
\end{abstract}

Keywords: Mixture Distributions; Rayleigh Distribution; Generalized Order Statistics; Record Values; MCMC

\section{Introduction}

The concept of generalized order statistics GOS was introduced by [1] as random variables having certain joint density function, which includes as a special case the joint density functions of many models of ordered random variables, such as ordinary order statistics, ordinary record values, progressive Type-II censored order statistics and sequential order statistics, among others. The GOS have been considered extensively by many authors, some of them are [2-18].

In life testing, reliability and quality control problems, mixed failure populations are sometimes encountered. Mixture distributions comprise a finite or infinite number of components, possibly of different distributional types, that can describe different features of data. In recent years, the finite mixture of life distributions have to be of considerable interest in terms of their practical applications in a variety of disciplines such as physics, biology, geology, medicine, engineering and economics, among others. Some of the most important references that discussed different types of mixtures of distributions are [19-25].

Let the random variable $T$ follows Rayleigh lifetime model, its probability density function (PDF), cumulative distribution function (CDF) and reliability function (RF) are given below:

$$
\begin{aligned}
& h(t)=2 \theta t e^{-\theta t^{2}}, t>0,(\theta>0), \\
& H(t)=1-e^{-\theta t^{2}}, t>0,(\theta>0),
\end{aligned}
$$

$$
R(t)=e^{-\theta t^{2}},
$$

Also, the hazard rate function (HRF)

$$
\lambda(t)=2 \theta t,
$$

where $\lambda()=.h(.) / R($.$) .$

The cumulative distribution function (CDF), denoted by $H(t)$, of a finite mixture of $k$ components, denoted by $H_{j}(t), j=1, \cdots, k$ is given by

$$
H(t)=\sum_{j=1}^{k} p_{j} H_{j}(t),
$$

where, for $j=1, \cdots, k$ the mixing proportions $p_{j} \geq 0$ and $\sum_{j=1}^{k} p_{j}=1$. The case of $k=2$, in (5), is practical importance and so, we shall restrict our study to this case. In such case, the population consist of two sub-populations, mixed with proportions $p_{1}$ and $p_{2}=1-p_{1}$. In this paper, the components are assumed to be Rayleigh distribution whose PDF, CDF, RF and HRF are given, respectively, by

$$
\begin{gathered}
h(t)=p_{1} h_{1}(t)+p_{2} h_{2}(t) \\
H(t)=p_{1} H_{1}(t)+p_{2} H_{2}(t), \\
R(t)=p_{1} R_{1}(t)+p_{2} R_{2}(t), \\
\lambda(t)=h(t) / R(t),
\end{gathered}
$$

where, for $j=1,2$, the mixing proportions $p_{j}$ are such 
that $0 \leq p_{j} \leq 1, p_{1}+p_{2}=1$ and $h_{j}(t), H_{j}(t), R_{j}(t)$ are given from (1)-(3) after using $\theta_{j}$ instead of $\theta$.

Several authors have predicted future order statistics and records from homogeneous and heterogeneous populations that can be represented by single component distribution and finite mixtures of distributions, respectively. For more details, see [9,10,26].

Recently, a few of authors utilized the GOS's in Bayesian inference. Such authors are [7-9,18]. Bayesian inferences based on finite mixture distribution have been discussed by several authors such that: [23,24,27-33].

For Bayesian approach, the performance depends on the form of the prior distribution and the loss function assumed. A wide variety of loss functions have been developed in the literature to describe various types of loss structures. The balanced loss function was suggested by [34]. [35] introduced an extended class of the balanced loss function of the form

$$
\begin{aligned}
& L_{\Phi, \Omega, \delta_{0}}(\Psi(\theta), \delta) \\
& =\Omega \Upsilon(\theta) \Phi\left(\delta_{0}, \delta\right)+(1-\Omega) \Upsilon(\theta) \Phi(\Psi(\theta), \delta),
\end{aligned}
$$

where $\Upsilon($.$) is a suitable positive weight function and$ $\Phi(\Psi(\theta), \delta)$ is an arbitrary loss function when estimateing $\Psi(\theta)$ by $\delta$. The parameter $\delta_{0}$ is a chosen prior estimator of $\Psi(\theta)$, obtained for instance from the criterion of maximum likelihood (ML), least squares or unbiasedness among others. They give a general Bayesian connection between the case of $\Omega>0$ and $\Omega=0$ where $0 \leq \Omega<1$.

Suppose that $T_{1 ; n, \tilde{m}, k}, T_{2 ; n, \tilde{m}, k}, \cdots, T_{r ; n, \tilde{m}, k}, k>0$, $\tilde{m}=\left(m_{1}, \cdots, m_{r-1}\right) \in R^{r-1}, m_{1}, \cdots, m_{r-1} \in R$ are the first $r$ (out of $n$ ) GOS drawn from the mixture of two Rayleigh MTR distribution. The likelihood function (LF) is given in [1], for $-\infty<t_{1}<\cdots<t_{r}<\infty$ by

$$
L(\theta \mid t)=C_{r-1}\left[\prod_{i=1}^{r-1}\left[R\left(t_{i}\right)\right]^{m_{i}} h\left(t_{i}\right)\right]\left[R\left(t_{r}\right)\right]^{\gamma_{r}-1} h\left(t_{r}\right),
$$

where $t=\left(t_{1}, \cdots, t_{r}\right), \theta \in \Theta, \Theta$ is the parameter space, and

$$
\begin{aligned}
& C_{r-1}=\prod_{i=1}^{r} \gamma_{i}, \gamma_{i}=k+n-i+M_{i}>0, \\
& M_{i}=\sum_{v=i}^{n-1} \nu=i .
\end{aligned}
$$

where $h\left(t_{i}\right)$ and $R\left(t_{i}\right)$ are given, respectively, by (5) and (7).

The purpose of this paper is to obtained the maximum likelihood prediction (point and interval) and the Bayes prediction (point and interval) in the case of one-sample scheme and two-sample scheme. The point predictors are obtained based on balanced square error loss (BSEL) function and the balanced LINEX (BLINEX) loss function. We used ML to estimate the parameters, $p$ and $\theta_{j}$ of the MTR distribution based on GOS. The conjugate prior is assumed to carry out the Bayesian analysis. The results are specialized to the upper record values. The rest of the article is organized as follows. Section 2 deals with the derivation of the maximum likelihood estimators of the involved parameters. Sections 3 and 4, deals with studying the maximum likelihood (point and interval) and the Bayes prediction (point and interval) in the case of one-sample scheme and two-sample scheme. In Section 5, the numerical computations results are presented and the concluding remarks.

\section{Maximum Likelihood Estimation (MLE)}

Substituting (6), (7) in (11), the LF takes the form

$$
\begin{aligned}
L(\theta \mid t) \propto \prod_{i=1}^{r-1}\left[p_{1} R_{1}\left(t_{i}\right)+p_{2} R_{2}\left(t_{i}\right)\right]^{m_{i}} \\
\prod_{i=1}^{r}\left[p_{1} h_{1}\left(t_{i}\right)+p_{2} h_{2}\left(t_{i}\right)\right] \\
\quad\left[p_{1} R_{1}\left(t_{r}\right)+p_{2} R_{2}\left(t_{r}\right)\right]^{\gamma_{r}-1} .
\end{aligned}
$$

Take the logarithm of (13), we have

$$
\begin{aligned}
l(\theta) \equiv & \ln L(\theta \mid t) \propto \sum_{i=1}^{r-1} m_{i} \ln \left[p_{1} R_{1}\left(t_{i}\right)+p_{2} R_{2}\left(t_{i}\right)\right] \\
& +\sum_{i=1}^{r} \ln \left[p_{1} h_{1}\left(t_{i}\right)+p_{2} h_{2}\left(t_{i}\right)\right] \\
& +\left(\gamma_{r}-1\right) \ln \left[p_{1} R_{1}\left(t_{r}\right)+p_{2} R_{2}\left(t_{r}\right)\right]
\end{aligned}
$$

where $p_{1}=p, p_{2}=1-p$. Differentiating (14) with respect to the parameters $p$ and $\theta_{j}$ and equating to zero gives the following likelihood equations

$$
\left.\begin{array}{r}
\frac{\partial l}{\partial p}=\sum_{i=1}^{r-1} m_{i} \vartheta^{*}\left(t_{i}\right)+\sum_{i=1}^{r} \vartheta\left(t_{i}\right)+\left(\gamma_{r}-1\right) \vartheta^{*}\left(t_{r}\right)=0, \\
\frac{\partial l}{\partial \theta_{j}}=p_{j} \sum_{i=1}^{r} \xi_{j}\left(t_{i}\right) \psi_{j}\left(t_{i}\right) \\
-\sum_{i=1}^{r-1} m_{i} \psi_{j}^{*}\left(t_{i}\right)-\left(\gamma_{r}-1\right) \psi_{j}^{*}\left(t_{r}\right)=0 \\
j=1,2
\end{array}\right\}
$$

where, for $j=1,2$

$$
\left.\begin{array}{r}
\vartheta\left(t_{i}\right)=\frac{h_{1}\left(t_{i}\right)-h_{2}\left(t_{i}\right)}{h\left(t_{i}\right)}, \vartheta^{*}\left(t_{i}\right)=\frac{R_{1}\left(t_{i}\right)-R_{2}\left(t_{i}\right)}{R\left(t_{i}\right)}, \\
\psi_{j}\left(t_{i}\right)=\frac{h_{j}\left(t_{i}\right)}{h\left(t_{i}\right)}, \psi_{j}^{*}\left(t_{i}\right)=\frac{t_{i}^{2} R_{j}\left(t_{i}\right)}{R\left(t_{i}\right)}, \\
\xi_{j}\left(t_{i}\right)=\left[\frac{1}{\theta_{j}}-t_{i}^{2}\right]
\end{array}\right\}
$$


Equations (15) do not yield explicit solutions for $p$ and $\theta_{j}, j=1,2$ and have to be solved numerically to obtain the ML estimates of the three parameters. Newton-Raphson iteration is employed to solve (15).

Remark: The parameters of the components are assumed to be distinct, so that the mixture is identifiable. For the concept of identifiability of finite mixtures and examples, see [19,36,37].

\section{Prediction in Case of One-Sample Scheme}

Based on the informative $T_{1 ; n, \tilde{m}, k}, \cdots, T_{r ; n, \tilde{m}, k}$ GOS's from the MTR distribution, for the remaining unobserved future $(n-r)$ components, let $T_{s ; n, \tilde{m}, k}, s=r+1, r+$ $2, \cdots, n$ denote the future lifetime of the $s^{\text {th }}$ component to fail, $1 \leq s \leq(n-r)$, the maximum Likelihood prediction (point (MLPP) and interval (MLPI)), Bayesian prediction (point (BPP) and interval (BPI)) can be obtained.

The conditional PDF of $T_{s} \equiv T_{s: n, \tilde{m}, k}$ given that the $T_{r} \equiv T_{r ; n, \tilde{m}, k}$ components that had already failed is

$$
\begin{aligned}
& k^{*}\left(t_{s} \mid t_{r}\right)=\frac{k^{s-r}}{(s-r-1) !}\left[\ln R\left(t_{r}\right)-\ln R\left(t_{s}\right)\right]^{s-r-1} \\
& \times\left[R\left(t_{s}\right)\right]^{k-1}\left[R\left(t_{r}\right)\right]^{-k} h\left(t_{s}\right), m=-1, \\
& \frac{C_{s-1}}{(m+1)^{s-r-1}(s-r-1) ! C_{r-1}}\left[R\left(t_{r}\right)^{m+1}-R\left(t_{s}\right)^{m+1}\right]^{s-r-1} \\
& \times\left[R\left(t_{s}\right)\right]^{\gamma_{s}-1}\left[R\left(t_{r}\right)\right]^{-\gamma_{r+1}} h\left(t_{s}\right), m \neq-1,
\end{aligned}
$$

In the case when $m=-1$, substituting (6) and (7) in (17), the conditional PDF takes the form

$$
\begin{aligned}
k_{1}^{*}\left(t_{s} \mid \theta_{j}\right) & \propto\left[p_{1} R_{1}\left(t_{s}\right)+p_{2} R_{2}\left(t_{s}\right)\right]^{k-1}\left[p_{1} R_{1}\left(t_{r}\right)+p_{2} R_{2}\left(t_{r}\right)\right]^{-k} \\
\times & {\left[\ln \left[p_{1} R_{1}\left(t_{r}\right)+p_{2} R_{2}\left(t_{r}\right)\right]\right.} \\
& \left.\left.-\ln \left[p_{1} R_{1}\left(t_{s}\right)+p_{2} R_{2}\left(t_{s}\right)\right]\right]^{s-r-1}\right] \\
& {\left[p_{1} h_{1}\left(t_{s}\right)+p_{2} h_{2}\left(t_{s}\right)\right], t_{s}>t_{r} . }
\end{aligned}
$$

And in the case when $m \neq-1$, substituting (6) and (7) in (17), the conditional PDF takes the form

$$
\begin{aligned}
k_{2}^{*}\left(t_{s} \mid \theta_{j}\right) \propto & {\left[p_{1} R_{1}\left(t_{s}\right)+p_{2} R_{2}\left(t_{s}\right)\right]^{\gamma_{s}-1} } \\
& {\left[p_{1} R_{1}\left(t_{r}\right)+p_{2} R_{2}\left(t_{r}\right)\right]^{-\gamma_{r+1}} } \\
& {\left[\left[p_{1} R_{1}\left(t_{r}\right)+p_{2} R_{2}\left(t_{r}\right)\right]^{m+1}\right.} \\
& \left.-\left[p_{1} R_{1}\left(t_{s}\right)+p_{2} R_{2}\left(t_{s}\right)\right]^{m+1}\right]^{s-r-1} \\
& {\left[p_{1} h_{1}\left(t_{s}\right)+p_{2} h_{2}\left(t_{s}\right)\right], t_{s}>t_{r} . }
\end{aligned}
$$

In the following, we considered two cases: the first is when the mixing proportion $p$ is known and the second is when the two parameters $\theta$ and $p$ are assumed to be unknown.

\subsection{Prediction When $p$ Is Known}

In this section we estimate $\theta_{1}$ and $\theta_{2}$, assuming that the mixing proportion, $p_{1}$ and $p_{2}$ are known.

\subsubsection{Maximum Likelihood Prediction}

Maximum likelihood prediction can be obtain using (18) and (19) by replacing the shape parameters $\theta_{1}$ and $\theta_{2}$ by $\hat{\theta}_{1(M L)}$ and $\hat{\theta}_{2(M L)}$ which is obtained from (15).

1) Interval prediction

The MLPI for any future observation $t_{s}, s=r+1, r+$ $2, \cdots, n$ can be obtained by

$$
\begin{aligned}
\operatorname{Pr}\left[t_{s} \geq v \mid \boldsymbol{t}\right] & =\int_{v}^{\infty} k_{1}^{*}\left(t_{s} \mid \hat{\theta}_{1(M L)}, \hat{\theta}_{2(M L)}\right) \mathrm{d} t_{s}, m=-1, \\
& =\int_{v}^{\infty} k_{2}^{*}\left(t_{s} \mid \hat{\theta}_{1(M L)}, \hat{\theta}_{2(M L)}\right) \mathrm{d} t_{s}, m \neq 1 .
\end{aligned}
$$

A $(1-\tau) \times 100 \%$ MLPI $(L, U)$ of the future observation $t_{s}$ is given by solving the following two nonlinear equations

$$
\operatorname{Pr}\left[t_{s} \geq L(t) \mid t\right]=1-\frac{\tau}{2}, \operatorname{Pr}\left[t_{s} \geq U(t) \mid t\right]=\frac{\tau}{2} .
$$

2) Point prediction

The MLPP for any future observation $t_{s}, s=r+1, r$ $+2, \cdots, n$ can be obtained by replacing the shape parameters $\theta_{1}$ and $\theta_{2}$ by $\hat{\theta}_{1(M L)}$ and $\hat{\theta}_{2(M L)}$ which, obtained from (15)

$$
\begin{aligned}
\hat{t}_{s(M L)}=E\left(t_{s}\right) & =\int_{v}^{\infty} k_{1}^{*}\left(t_{s} \mid \hat{\theta}_{1(M L)}, \hat{\theta}_{2(M L)}\right) \mathrm{d} t_{s}, m=-1, \\
& =\int_{v}^{\infty} k_{2}^{*}\left(t_{s} \mid \hat{\theta}_{1(M L)}, \hat{\theta}_{2(M L)}\right) \mathrm{d} t_{s}, m \neq 1 .
\end{aligned}
$$

\subsubsection{Bayesian Prediction}

When the mixing proportion, $p$ is known. Let the parameters $\theta_{j}, j=1,2$ have a gamma prior distribution with PDF

$$
\pi\left(\theta_{j}\right)=\frac{1}{\Gamma\left(v_{j}\right)} \beta_{j}^{v_{j}} \theta_{j}^{v_{j}-1} e^{-\beta_{j} \theta_{j}},\left(\theta_{j}, v_{j}, \beta_{j}>0\right) .
$$

These are chosen since they are the conjugate priors for the individual parameters. The joint prior density function of $\theta=\left(\theta_{1}, \theta_{2}\right)$ is given by

$$
\begin{aligned}
& \pi(\theta)=\pi_{1}\left(\theta_{1}\right) \pi_{2}\left(\theta_{2}\right), \\
& \pi(\theta) \propto \prod_{j=1}^{2} \theta_{j}^{\nu_{j}-1} e^{-\sum_{j=1}^{2} \beta_{j} \theta_{j}},
\end{aligned}
$$

where $j=1,2, \theta_{j}>0,\left(v_{j}, \beta_{j}\right)>0$. 
It then follows, from (13) and (24), that the joint posterior density function is given by

$$
\begin{aligned}
\pi_{1}^{*}(\theta \mid t)= & A_{1} \prod_{j=1}^{2} \theta_{j}^{v_{j}-1} e^{-\sum_{j=1}^{2} \beta_{j} \theta_{j_{j}}} \\
& \prod_{i=1}^{r-1}\left[p_{1} R_{1}\left(t_{i}\right)+p_{2} R_{2}\left(t_{i}\right)\right]^{m_{i}} \\
& \prod_{i=1}^{r}\left[p_{1} h_{1}\left(t_{i}\right)+p_{2} h_{2}\left(t_{i}\right)\right] \\
& \times\left[p_{1} R_{1}\left(t_{r}\right)+p_{2} R_{2}\left(t_{r}\right)\right]^{\gamma_{r}-1}
\end{aligned}
$$

where

$$
A_{1}^{-1}=\int_{\theta} \pi(\theta) L(\theta \mid t) \mathrm{d} \theta .
$$

The Bayes predictive density function can be obtained using (18), (19) and (25) as follow:

$$
\begin{aligned}
& Q_{1}^{*}\left(t_{s} \mid t\right) \propto \int_{0}^{\infty} \pi_{1}^{*}(\theta \mid t) k_{1}^{*}\left(t_{s} \mid \theta_{j}\right) \mathrm{d} \theta, m=-1, \\
& Q_{1}^{*}\left(t_{s} \mid t\right) \propto \int_{0}^{\infty} \pi_{1}^{*}(\theta \mid t) k_{2}^{*}\left(t_{s} \mid \theta_{j}\right) \mathrm{d} \theta, m \neq-1 .
\end{aligned}
$$

1) Interval prediction

Bayesian prediction interval, for the future observation $T_{s: n, \hat{m}, k}, \quad s=r+1, r+2, \cdots, n$ can be computed by approximated $Q_{1}^{*}\left(t_{s} \mid \boldsymbol{t}\right)$ using the MCMC algorithm, see $[17,24]$, using the form

$$
Q_{1}^{*}\left(t_{s} \mid t\right) \cong \frac{\sum_{i=1}^{\mu} k^{*}\left(t_{s} \mid \theta_{j}^{i}\right)}{\sum_{i=1}^{\mu} \int_{t_{r}}^{\infty} k^{*}\left(t_{s} \mid \theta_{j}^{i}\right) \mathrm{d} t_{s}},
$$

where $\mu$ is the number of generated parameters and $\theta_{j}^{i}, \quad i=1,2,3, \cdots, \mu$. They are generated from the posterior density function (25) using Gibbs sampler and Metropolis-Hastings techniques, for more details see [38].

A $(1-\tau) \times 100 \%$ BPI $(L, U)$ of the future observation $t_{s}$ is given by solving the following two nonlinear equations

$$
\begin{aligned}
& \frac{\sum_{i=1}^{\mu} \int_{L}^{\infty} k^{*}\left(t_{s} \mid \theta_{j}^{i}\right) \mathrm{d} t_{s}}{\sum_{i=1}^{\mu} \int_{t_{r}}^{\infty} k^{*}\left(t_{s} \mid \theta_{j}^{i}\right) \mathrm{d} t_{s}}=1-\frac{\tau}{2}, \\
& \frac{\sum_{i=1}^{\mu} \int_{U}^{\infty} k^{*}\left(t_{s} \mid \theta_{j}^{i}\right) \mathrm{d} t_{s}}{\sum_{i=1}^{\mu} \int_{t_{r}}^{\infty} k^{*}\left(t_{s} \mid \theta_{j}^{i}\right) \mathrm{d} t_{s}}=\frac{\tau}{2} .
\end{aligned}
$$

Numerical methods are generally necessary to solve the above two equations to obtain $L$ and $U$ for a given $\tau$.

2) Point prediction

a) BPP for the future observation $t_{s}$ based on BSEL function can be obtained using

$$
\tilde{t}_{s(B S)}=\Omega \tilde{t}_{s(M L)}+(1-\Omega) E\left(t_{s} \mid t\right),
$$

where $\tilde{t}_{s(M L)}$ is the ML prediction for the future obser- vation $t_{s}$ which can be obtained using (22) and $E\left(t_{s} \mid \boldsymbol{t}\right)$ can be obtained using

$$
E\left(t_{s} \mid t\right)=\int_{t_{r}}^{\infty} t_{s} \mathrm{Q}_{1}^{*}\left(t_{s} \mid t\right) \mathrm{d} t_{s} .
$$

b) BPP for the future observation $t_{s}$ based on BLINX loss function can be obtained using

$$
\tilde{t}_{s(B L)}=-\frac{1}{a} \ln \left[\Omega e^{-a \tilde{t}_{s(M L)}}+(1-\Omega) E\left(e^{-a t_{s}} \mid t\right)\right],
$$

where $\tilde{t}_{s(M L)}$ is the ML prediction for the future observation $t_{s}$ which can be obtained using (22) and $E\left(e^{-a t_{s}} \mid \boldsymbol{t}\right)$ can be obtained using

$$
E\left(e^{-a t_{s}} \mid t\right)=\int_{t_{r}}^{\infty} e^{-a t_{s}} Q_{1}^{*}\left(t_{s} \mid t\right) \mathrm{d} t_{s} .
$$

\subsection{Prediction When $\boldsymbol{p}$ and $\boldsymbol{\theta}_{\boldsymbol{j}}$ Are Unknown}

When both of the two parameters the mixing proportion $p_{j}$ and $\theta_{j}, j=1,2$, are assumed to be unknown.

\subsubsection{Maximum Likelihood Prediction}

Maximum likelihood prediction can be obtain using (18) and (19) by replacing the parameters $p, \theta_{1}$ and $\theta_{2}$ by $\hat{p}_{(M L)}, \hat{\theta}_{1(M L)}$ and $\hat{\theta}_{2(M L)}$ which we obtained using (15).

1) Interval prediction

The MLPI for any future observation $t_{s}, s=r+1, r$ $+2, \cdots, n$ can be obtained by

$$
\begin{aligned}
\operatorname{Pr}\left[t_{s} \geq v \mid t\right]= & \int_{v}^{\infty} k_{1}^{*}\left(t_{s} \mid \hat{p}_{(M L)}, \hat{\theta}_{1(M L)}, \hat{\theta}_{2(M L)}\right) \mathrm{d} t_{s}, m=-1, \\
& =\int_{v}^{\infty} k_{2}^{*}\left(t_{s} \mid \hat{p}_{(M L)}, \hat{\theta}_{1(M L)}, \hat{\theta}_{2(M L)}\right) \mathrm{d} t_{s}, m \neq 1 .
\end{aligned}
$$

A $(1-\tau) \times 100 \%$ MLPI $(L, U)$ of the future observation $t_{s}$ is given by solving the following two nonlinear Equations (21).

2) Point prediction

The MLPP for any future observation $t_{s}, \quad s=r+1, r$ $+2, \cdots, n$ can be obtained by replacing the shape parameters $p, \theta_{1}$ and $\theta_{2}$ by $\hat{p}_{(M L)}, \hat{\theta}_{1(M L)}$ and $\hat{\theta}_{2(M L)}$ which, obtained from (15).

$$
\begin{aligned}
\hat{t}_{s(M L)}=E\left(t_{s}\right) & =\int_{t}^{\infty} t_{s} k_{1}^{*}\left(t_{s} \mid \hat{p}_{(M L)}, \hat{\theta}_{1(M L)}, \hat{\theta}_{2(M L)}\right) \mathrm{d} t_{s}, m=-1, \\
& =\int_{t}^{\infty} t_{s} k_{2}^{*}\left(t_{s} \mid \hat{p}_{(M L)}, \hat{\theta}_{1(M L)}, \hat{\theta}_{2(M L)}\right) \mathrm{d} t_{s}, m \neq 1 .
\end{aligned}
$$

\subsubsection{Bayesian Prediction}

Let $p_{j}$ and $\theta_{j}, j=1,2$, are independent random variables such that $p \sim \operatorname{Beta}\left(b_{1}, b_{2}\right)$ and for $j=1,2, \theta_{j}$ to follow an inverted gamma prior distribution with PDF 


$$
p\left(\theta_{j}\right)=\frac{1}{\Gamma\left(\alpha_{j}\right)}\left(\frac{\alpha_{j}}{\beta_{j}}\right)^{\alpha_{j}} \theta_{j}^{\alpha_{j}-1} e^{-\frac{\alpha_{j}}{\beta_{j}} \theta_{j}},\left(\theta_{j}, \alpha_{j}, \beta_{j}>0\right) .
$$

A joint prior density function of $\theta=\left(p, \theta_{1}, \theta_{2}\right)$ is then given by

$$
\begin{aligned}
& \pi(\theta)=\pi_{1}(p) \pi_{2}\left(\theta_{1}\right) \pi_{3}\left(\theta_{2}\right), \\
& \pi(\theta) \propto p_{1}^{b_{1}-1} p_{2}^{b_{2}-1} \prod_{j=1}^{2} \theta_{j}^{\alpha_{j}-1} e^{-\sum_{j=1}^{2} \frac{\alpha_{j}}{\beta_{j}} \theta_{j}},
\end{aligned}
$$

where $0<p_{1}<1, p_{2}=1-p_{1}$ and for $j=1,2 \theta_{j}>0$, $\left(b_{j}, \alpha_{j}, \beta_{j}\right)>0$.

Using the likelihood function (13) and the prior density function (38), the posterior density function will be in the form

$$
\begin{aligned}
& \pi_{2}^{*}(p, \theta \mid t) \\
& =A_{2} p_{1}^{b_{1}-1} p_{2}^{b_{2}-1} \prod_{j=1}^{2} \theta_{j}^{\alpha_{j}-1} e^{-\sum_{j=1}^{2} \frac{\alpha_{j}}{\beta_{j}} \theta_{j}} \\
& \prod_{i=1}^{r-1}\left[p_{1} R_{1}\left(t_{i}\right)+p_{2} R_{2}\left(t_{i}\right)\right]^{m_{i}} \\
& \prod_{i=1}^{r}\left[p_{1} h_{1}\left(t_{i}\right)+p_{2} h_{2}\left(t_{i}\right)\right]\left[p_{1} R_{1}\left(t_{r}\right)+p_{2} R_{2}\left(t_{r}\right)\right]^{\gamma_{r}-1},
\end{aligned}
$$

where

$$
A_{2}^{-1}=\int_{\theta} \pi(\theta) L(\theta \mid t) \mathrm{d} \theta
$$

The Bayes prediction density function of $T_{s} \equiv T(s, n, m, k)$ can be obtained, see [39], by

$$
\begin{aligned}
& Q_{2}^{*}\left(t_{s} \mid t\right) \\
= & \int_{0}^{\infty} \int_{0}^{1} \pi_{2}^{*}(p, \theta \mid t) k_{1}^{*}\left(t_{s} \mid \theta_{j}, p\right) \mathrm{d} p \mathrm{~d} \theta, m-1, \\
& \int_{0}^{\infty} \int_{0}^{1} \pi_{2}^{*}(p, \theta \mid t) k_{2}^{*}\left(t_{s} \mid \theta_{j}, p\right) \mathrm{d} p \mathrm{~d} \theta, m=-1 .
\end{aligned}
$$

1) Interval prediction

Bayesian prediction interval, for the future observation $T_{s ; n, \tilde{m}, k}, \quad s=r+1, r+2, \cdots, n$ can be computed by approximated $Q_{2}^{*}\left(t_{s} \mid t\right)$ using the MCMC algorithm, see [24], using the form

$$
Q_{2}^{*}\left(t_{s} \mid t\right) \cong \frac{\sum_{i=1}^{\mu} k^{*}\left(t_{s} \mid \theta_{j}^{i}, p^{i}\right)}{\sum_{i=1}^{\mu} \int_{t_{r}}^{\infty} k^{*}\left(t_{s} \mid \theta_{j}^{i}, p^{i}\right) \mathrm{d} t_{s}},
$$

where $\theta_{j}^{i}, p^{i}, i=1,2,3, \cdots, \mu$ are generated from the posterior density function (39) using Gibbs sampler and Metropolis-Hastings techniques. A $(1-\tau) \times 100 \%$ BPI $(L, U)$ of the future observation $t_{s}$ is given by solving the following two nonlinear equations

$$
\frac{\sum_{i=1}^{\mu} \int_{L}^{\infty} k^{*}\left(t_{s} \mid \theta_{j}^{i}, p^{i}\right) \mathrm{d} t_{s}}{\sum_{\mu}^{i=1} \int_{t_{r}}^{\infty} k^{*}\left(t_{s} \mid \theta_{j}^{i}, p^{i}\right) \mathrm{d} t_{s}}=1-\frac{\tau}{2},
$$

$$
\frac{\sum_{i=1}^{\mu} \int_{U}^{\infty} k^{*}\left(t_{s} \mid \theta_{j}^{i}, p^{i}\right) \mathrm{d} t_{s}}{\sum_{\mu}^{i=1} \int_{t_{r}}^{\infty} k^{*}\left(t_{s} \mid \theta_{j}^{i}, p^{i}\right) \mathrm{d} t_{s}}=\frac{\tau}{2},
$$

Numerical methods are generally necessary to solve the above two equations to obtain $L$ and $U$ for a given $\tau$.

1) Point prediction

BPP for the future observation $t_{s}$ based on BSEL function can be obtained using

$$
\tilde{t}_{s(B S)}=\Omega \tilde{t}_{s(M L)}+(1-\Omega) E\left(t_{s} \mid t\right),
$$

where $\tilde{t}_{s(M L)}$ is the ML prediction for the future observation $t_{s}$ which can be obtained using (36) and $E\left(t_{s} \mid \boldsymbol{t}\right)$ can be obtained using

$$
E\left(t_{s} \mid t\right)=\int_{t_{r}}^{\infty} t_{s} Q_{2}^{*}\left(t_{s} \mid t\right) \mathrm{d} t_{s} .
$$

2) BPP for the future observation $t_{s}$ based on BLINX loss function can be obtained using

$$
\tilde{t}_{s(B L)}=-\frac{1}{a} \ln n\left[\Omega e^{-a \tilde{t}_{s(M L)}}+(1-\Omega) E\left(e^{-a t_{s}} \mid t\right)\right],
$$

where $\tilde{t}_{s(M L)}$ is the ML prediction for the future observation $t_{s}$ which can be obtained using (36) and $E\left(e^{-a t_{s}} \mid t\right)$ can be obtained using

$$
E\left(e^{-a t_{s}} \mid t\right)=\int_{t_{r}}^{\infty} e^{-a t_{s}} Q_{2}^{*}\left(t_{s} \mid t\right) \mathrm{d} t_{s} .
$$

\section{Prediction in Case of Two-Sample Scheme}

Based on the informative $T_{1 ; n, \tilde{m}, k}, T_{2 ; n, \tilde{m}, k}, \cdots, T_{r ; n, \tilde{m}, k}$ GOS drawn from the MTR distribution and let $Y_{1}<\cdots<Y_{N}$, where $Y_{i} \equiv Y_{i, N, M, K}, i=1,2, \cdots, N, M>0, K>0$ be a second independent generalized ordered random sample (of size $N$ ) of future observations from the same distribution. We want to predict any future (unobserved) GOS $Y_{b} \equiv Y_{b ; N, M, K}, b=1,2, \cdots, N$, in the future sample of size $N$. The PDF of $Y_{b}, 1 \leq b \leq N$ given the vector of parameters $\theta$, is:

$$
\begin{aligned}
& G^{*}\left(y_{b} \mid \theta\right) \propto \\
& {\left[R\left(y_{b}\right)\right]^{\gamma_{b}^{*}-1} h\left(y_{b}\right) \sum_{j=0}^{b-1} \omega_{j}^{b}\left[R\left(y_{b}\right)\right]^{j(M+1)}, M \neq-1,} \\
& {\left[R\left(y_{b}\right)\right]^{K-1}\left[\ln R\left(y_{b}\right)\right]^{b-1} h\left(y_{b}\right), M=-1,}
\end{aligned}
$$

where $\omega_{j}^{b}=(-1)^{j}\left(\begin{array}{c}b-1 \\ j\end{array}\right)$ and $\gamma_{j}^{\star}=K+(N-j)(M+1)$

Substituting from (6) and (7) in (49), we have:

$$
\begin{aligned}
& G_{1}^{*}\left(y_{b} \mid \theta\right) \propto \\
& {\left[p_{1} R_{1}\left(y_{b}\right)+p_{2} R_{2}\left(y_{b}\right)\right]^{\gamma_{b}^{*}-1}\left[p_{1} h_{1}\left(y_{b}\right)+p_{2} h_{2}\left(y_{b}\right)\right]} \\
& \sum_{j=0}^{b-1} \omega_{j}^{b}\left[p_{1} R_{1}\left(y_{b}\right)+p_{2} R_{2}\left(y_{b}\right)\right]^{j(M+1)}, M \neq-1,
\end{aligned}
$$




$$
\begin{aligned}
& G_{2}^{*}\left(y_{b} \mid \theta\right) \propto \\
& {\left[p_{1} R_{1}\left(y_{b}\right)+p_{2} R_{2}\left(y_{b}\right)\right]^{K-1}\left[p_{1} h_{1}\left(y_{b}\right)+p_{2} h_{2}\left(y_{b}\right)\right]} \\
& {\left[\ln \left[p_{1} R_{1}\left(y_{b}\right)+p_{2} R_{2}\left(y_{b}\right)\right]\right]^{(b-1)}, M=-1,}
\end{aligned}
$$

\subsection{Prediction When $P$ Is Known}

\subsubsection{Maximum Likelihood Prediction}

Maximum likelihood prediction can be obtain using (50) and (51) by replacing the shape parameters $\theta_{1}$ and $\theta_{2}$ by $\hat{\theta}_{1(M L)}$ and $\hat{\theta}_{2(M L)}$

1) Interval prediction

The MLPI for any future observation $y_{b}, 1 \leq b \leq N$ can be obtained by

$$
\begin{aligned}
\operatorname{Pr}\left[t_{s} \geq v \mid \boldsymbol{t}\right] & =\int_{V}^{\infty} G_{1}^{*}\left(y_{b} \mid \hat{\theta}_{1(M L)}, \hat{\theta}_{2(M L)}\right) \mathrm{d} y_{b}, M \neq-1, \\
& =\int_{v}^{\infty} G_{2}^{*}\left(y_{b} \mid \hat{\theta}_{1(M L)}, \hat{\theta}_{2(M L)}\right) \mathrm{d} y_{b}, M=1 .
\end{aligned}
$$

A $(1-\tau) \times 100 \%$ MLPI $(L, U)$ of the future observation $y_{b}$ is given by solving the following two nonlinear equations

$$
\operatorname{Pr}\left[y_{b} \geq L(t) \mid \boldsymbol{t}\right]=1-\frac{\tau}{2}, \operatorname{Pr}\left[y_{b} \geq U(t) \mid t\right]=\frac{\tau}{2}
$$

2) Point prediction

The MLPP for any future observation $y_{b}$ can be obtained by replacing the shape parameters $\theta_{1}$ and $\theta_{2}$ by $\hat{\theta}_{1(M L)}$ and $\hat{\theta}_{2(M L)}$

$$
\begin{aligned}
\hat{y}_{b(M L)} & =E\left(y_{b} \mid \boldsymbol{t}\right) \\
& =\int_{0}^{\infty} y_{b} G_{1}^{*}\left(y_{b} \mid \hat{\theta}_{1(M L)}, \hat{\theta}_{2(M L)}\right) \mathrm{d} y_{b}, M \neq-1, \\
& =\int_{0}^{\infty} y_{b} G_{2}^{*}\left(y_{b} \mid \hat{\theta}_{1(M L)}, \hat{\theta}_{2(M L)}\right) \mathrm{d} y_{b}, M=1 .
\end{aligned}
$$

\subsubsection{Bayesian Prediction}

The predictive density function of $Y_{b}, 1 \leq b \leq N$ is given by:

$$
\Psi^{*}\left(y_{b} \mid \boldsymbol{t}\right)=\int_{0}^{\infty} G^{*}\left(y_{b} \mid \theta\right) \pi_{1}^{*}(\theta \mid t) \mathrm{d} \theta, y_{b}>0,
$$

where for $M \neq-1$ and $m \neq-1$

$$
\begin{aligned}
& \Psi^{*}\left(y_{b} \mid t\right) \\
& =\int_{0}^{\infty} \pi_{1}^{*}(\theta \mid t) G^{*}\left(y_{b} \mid \theta\right) \mathrm{d} \theta \\
& \propto \prod_{j=1}^{2} \theta_{j}^{v_{j}-1} e^{-\sum_{j=1}^{2} \beta_{j} \theta_{j}} \prod_{i=1}^{r-1}\left[p_{1} R_{1}\left(t_{i}\right)+p_{2} R_{2}\left(t_{i}\right)\right]^{m_{i}} \\
& \times \prod_{i=1}^{r}\left[p_{1} h_{1}\left(t_{i}\right)+p_{2} h_{2}\left(t_{i}\right)\right]\left[p_{1} R_{1}\left(t_{r}\right)+p_{2} R_{2}\left(t_{r}\right)\right]^{\gamma_{r}-1} \\
& \times\left[p_{1} R_{1}\left(y_{b}\right)+p_{2} R_{2}\left(y_{b}\right)\right]^{\gamma_{b}^{*}-1}\left[p_{1} h_{1}\left(y_{b}\right)+p_{2} h_{2}\left(y_{b}\right)\right] \\
& \sum_{j=0}^{b-1} \omega_{j}^{b}\left[p_{1} R_{1}\left(y_{b}\right)+p_{2} R_{2}\left(y_{b}\right)\right]^{j(M+1)} \mathrm{d} \theta .
\end{aligned}
$$

$$
\begin{aligned}
& \text { Also, when } M=-1 \text { and } m=-1 \\
& \Psi_{2}^{*}\left(y_{b} \mid t\right) \\
& =\int_{0}^{\infty} \pi_{1}^{*}(\theta \mid t) G_{2}^{*}\left(y_{b} \mid \theta\right) \mathrm{d} \theta \\
& \propto \prod_{j=1}^{2} \theta_{j}^{v_{j}-1} e^{-\sum_{j=1}^{2} \beta_{j} \theta_{j}} \prod_{i=1}^{r-1}\left[p_{1} R_{1}\left(t_{i}\right)+p_{2} R_{2}\left(t_{i}\right)\right]^{-1} \\
& \times \prod_{i=1}^{r}\left[p_{1} h_{1}\left(t_{i}\right)+p_{2} h_{2}\left(t_{i}\right)\right]\left[p_{1} R_{1}\left(t_{r}\right)+p_{2} R_{2}\left(t_{r}\right)\right]^{\gamma_{r}-1} \\
& \times\left[\ln \left[p_{1} R_{1}\left(y_{b}\right)+p_{2} R_{2}\left(y_{b}\right)\right]\right]^{b-1}\left[p_{1} h_{1}\left(y_{b}\right)+p_{2} h_{2}\left(y_{b}\right)\right] \\
& {\left[p_{1} R_{1}\left(y_{b}\right)+p_{2} R_{2}\left(y_{b}\right)\right]^{K-1} \mathrm{~d} \theta .}
\end{aligned}
$$

1) Interval prediction

Bayesian prediction interval, for the future observation $Y_{b}, 1 \leq b \leq N$, can be computed using (56) and (57) which can be approximated using MCMC algorithm by the form

$$
\Psi^{*}\left(y_{b} \mid t\right) \cong \frac{\sum_{i=1}^{\mu} G^{*}\left(y_{b} \mid \theta_{j}^{i}\right)}{\sum_{i=1}^{\mu} \int_{0}^{\infty} G^{*}\left(y_{b} \mid \theta_{j}^{i}\right) \mathrm{d} y_{b}}
$$

where $\theta_{j}^{i}, i=1,2,3, \cdots, \mu$ are generated from the posterior density function (25) using Gibbs sampler and Metropolis-Hastings techniques.

A $(1-\tau) \times 100 \%$ BPI $(L, U)$ of the future observation $y_{b}$ is given by solving the following two nonlinear equations

$$
\begin{aligned}
& \frac{\sum_{i=1}^{\mu} \int_{L}^{\infty} G^{*}\left(y_{b} \mid \theta_{j}^{i}\right) \mathrm{d} y_{b}}{\sum_{\mu}^{i=1} \int_{0}^{\infty} G^{*}\left(y_{b} \mid \theta_{j}^{i}\right) \mathrm{d} y_{b}}=1-\frac{\tau}{2}, \\
& \frac{\sum_{i=1}^{\mu} \int_{U}^{\infty} G^{*}\left(y_{b} \mid \theta_{j}^{i}\right) \mathrm{d} y_{b}}{\sum_{\mu}^{i=1} \int_{0}^{\infty} G^{*}\left(y_{b} \mid \theta_{j}^{i}\right) \mathrm{d} y_{b}}=\frac{\tau}{2},
\end{aligned}
$$

Numerical methods such as Newton-Raphson are generally necessary to solve the above two nonlinear Equations (59) and (60), to obtain $L$ and $U$ for a given $\tau$.

2) Point prediction

a) BPP for the future observation $y_{b}$ based on BSEL function can be obtained using

$$
\tilde{y}_{b(B S)}=\Omega \hat{y}_{b(M L)}+(1-\Omega) E\left(y_{b} \mid t\right),
$$

where $\hat{y}_{b(M L)}$ is the ML prediction for the future observation $y_{b}$ which can be obtained using (54) and $E\left(y_{b} \mid t\right)$ can be obtained using

$$
E\left(y_{b} \mid t\right)=\int_{0}^{\infty} y_{b} \Psi^{*}\left(y_{b} \mid t\right) \mathrm{d} y_{b} .
$$

b) BPP for the future observation $y_{b}$ based on BLINX loss function can be obtained using 


$$
\tilde{y}_{b(B L)}=-\frac{1}{a} \ln \left[\Omega e^{-a \hat{y}_{b(M L)}}+(1-\Omega) E\left(e^{-a y_{b}} \mid t\right)\right],
$$

where $\hat{y}_{b(M L)}$ is the ML prediction for the future observation $y_{b}$ which can be obtained using (54) and $E\left(e^{-a y_{b}} \mid t\right)$ can be obtained using

$$
E\left(e^{-a y_{b}} \mid t\right)=\int_{0}^{\infty} e^{-a y_{b}} \Psi^{*}\left(y_{b} \mid t\right) \mathrm{d} y_{b} .
$$

\subsection{Prediction When $p$ and $\theta_{i}$ Are Unknown}

\subsubsection{Maximum Likelihood Prediction}

Maximum likelihood prediction can be obtain using (50) and (51) by replacing the parameters $p, \theta_{1}$ and $\theta_{2}$ by $\hat{p}_{(M L)}, \hat{\theta}_{1(M L)}$ and $\hat{\theta}_{2(M L)}$

1) Interval prediction

The maximum likelihood Interval prediction (MLIP) for any future observation $y_{b}, 1 \leq b \leq N$ can be obtained by

$$
\begin{aligned}
\operatorname{Pr}\left[t_{s} \geq v \mid \boldsymbol{t}\right] & =\int_{v}^{\infty} G_{1}^{*}\left(y_{b} \mid \hat{\theta}_{1(M L)}, \hat{\theta}_{2(M L)}, \hat{p}_{(M L)}\right) \mathrm{d} y_{b}, M \neq-1, \\
& =\int_{v}^{\infty} G_{2}^{*}\left(y_{b} \mid \hat{\theta}_{1(M L)}, \hat{\theta}_{2(M L)}, \hat{p}_{(M L)}\right) \mathrm{d} y_{b}, M=1 .
\end{aligned}
$$

A $(1-\tau) \times 100 \%$ MLPI $(L, U)$ of the future observation $y_{b}$ is given by solving the following two nonlinear equations

$$
\operatorname{Pr}\left[y_{b} \geq L(t) \mid t\right]=1-\frac{\tau}{2}, \operatorname{Pr}\left[y_{b} \geq U(t) \mid t\right]=\frac{\tau}{2}
$$

2) Point prediction

The MLPP for any future observation $y_{b}, 1 \leq b \leq N$ can be obtained by replacing the parameters $p, \theta_{1}$ and $\theta_{2}$ by $\hat{p}_{(M L)}, \hat{\theta}_{1(M L)}$ and $\hat{\theta}_{2(M L)}$

$$
\begin{aligned}
\hat{y}_{b(M L)} & =E\left(y_{b} \mid t\right) \\
& =\int_{0}^{\infty} y_{b} G_{1}^{*}\left(y_{b} \mid \hat{\theta}_{1(M L)}, \hat{\theta}_{2(M L)}, \hat{p}_{(M L)}\right) \mathrm{d} y_{b}, M \neq-1, \\
& =\int_{v}^{\infty} y_{b} G_{2}^{*}\left(y_{b} \mid \hat{\theta}_{1(M L)}, \hat{\theta}_{2(M L)}, \hat{p}_{(M L)}\right) \mathrm{d} y_{b}, M=-1 .
\end{aligned}
$$

\subsubsection{Bayesian Prediction}

The predictive density function of $y_{b}, 1 \leq b \leq N$ is given by:

$$
\Psi^{*}\left(y_{b} \mid \boldsymbol{t}\right)=\int_{0}^{\infty} \int_{0}^{1} G^{*}\left(y_{b} \mid p, \theta\right) \pi_{2}^{*}(\theta \mid \boldsymbol{t}) \mathrm{d} p \mathrm{~d} \theta, y_{b}>0
$$

where for $M \neq-1$ and $m \neq-1$

$$
\Psi_{1}^{*}\left(y_{b} \mid \boldsymbol{t}\right)=\int_{0}^{\infty} \int_{0}^{1} G_{1}^{*}\left(y_{b} \mid p, \theta\right) \pi_{2}^{*}(\theta \mid \boldsymbol{t}) \mathrm{d} p \mathrm{~d} \theta .
$$

Also, when $M=-1$ and $m=-1$

$$
\Psi_{2}^{*}\left(y_{b} \mid \boldsymbol{t}\right)=\int_{0}^{\infty} \int_{0}^{1} G_{2}^{*}\left(y_{b} \mid p, \theta\right) \pi_{2}^{*}(\theta \mid \boldsymbol{t}) \mathrm{d} p \mathrm{~d} \theta .
$$

\section{1) Interval prediction}

Bayesian prediction interval, for the future observation $y_{b}, 1 \leq b \leq N$, can be computed using (69) and (70) which can be approximated using MCMC algorithm by the form

$$
\Psi^{\star}\left(y_{b} \mid t\right) \cong \frac{\sum_{i=1}^{\mu} G^{*}\left(y_{b} \mid p^{i}, \theta_{j}^{i}\right)}{\sum_{i=1}^{\mu} \int_{0}^{\infty} G^{*}\left(y_{b} \mid p^{i}, \theta_{j}^{i}\right) d y_{b}}
$$

where $p^{i}, \theta_{j}^{i}, i=1,2, \cdots, \mu$ are generated from the posterior density function (39) using Gibbs sampler and Metropolis-Hastings techniques.

A $(1-\tau) \times 100 \%$ BPI $(L, U)$ of the future observation $y_{b}$ is given by solving the following two nonlinear equations

$$
\begin{aligned}
& \frac{\sum_{i=1}^{\mu} \int_{L}^{\infty} G^{*}\left(y_{b} \mid p^{i}, \theta_{j}^{i}\right) \mathrm{d} y_{b}}{\sum_{\mu}^{i=1} \int_{0}^{\infty} G^{*}\left(y_{b} \mid p^{i}, \theta_{j}^{i}\right) \mathrm{d} y_{b}}=1-\frac{\tau}{2}, \\
& \frac{\sum_{i=1}^{\mu} \int_{U}^{\infty} G^{*}\left(y_{b} \mid p^{i}, \theta_{j}^{i}\right) \mathrm{d} y_{b}}{\sum_{\mu}^{i=1} \int_{0}^{\infty} G^{*}\left(y_{b} \mid p^{i}, \theta_{j}^{i}\right) \mathrm{d} y_{b}}=\frac{\tau}{2},
\end{aligned}
$$

Numerical methods such as Newton-Raphson are necessary to solve the above two nonlinear equations (72) and (73), to obtain $L$ and $U$ for a given $\tau$.

2) Point prediction

a) BPP for the future observation $y_{b}$ based on BSEL function can be obtained using

$$
\tilde{y}_{b(B S)}=\Omega \hat{y}_{b(M L)}+(1-\Omega) E\left(y_{b} \mid t\right),
$$

where $\hat{y}_{b(M L)}$ is the ML prediction for the future observation $y_{b}$ which can be obtained using (67) and $E\left(y_{b} \mid \boldsymbol{t}\right)$

$$
E\left(y_{b} \mid t\right)=\int_{0}^{\infty} y_{b} \Psi^{\star}\left(y_{b} \mid t\right) \mathrm{d} y_{b} .
$$

2) BPP for the future observation $y_{b}$ based on BLINX loss function can be obtained using

$$
\tilde{y}_{b(B L)}=-\frac{1}{a} \ln \left[\Omega e^{-a \hat{y}_{b(M L)}}+(1-\Omega) E\left(e^{-a y_{b}} \mid t\right)\right],
$$

where $\hat{y}_{b(M L)}$ is the ML prediction for the future observation $y_{b}$ which can be obtained using (67) and $E\left(e^{-a y_{b}} \mid t\right)$ can be obtained using

$$
E\left(e^{-a y_{b}} \mid t\right)=\int_{0}^{\infty} e^{-a y_{b}} \Psi^{\star}\left(y_{b} \mid t\right) \mathrm{d} y_{b} .
$$

\section{Simulation Procedure}

In this subsection we will consider the upper record values which can be obtained from the GOS by taking $m=-1, k=1$ and $\gamma_{r}=1$. In this section, we will compute point and interval predictors of future upper record 
values in two cases, one sample and two sample prediction as following:

\subsection{One Sample Prediction}

The following steps are used to obtain ML prediction (point and interval) and Bayesian prediction (point and interval) for the remaining $(n-r)$ failure times

$T_{s} \equiv T_{s, n, \hat{m}, k}, s=r+1, r+2$

1) For given values of $p, \theta_{1}$ and $\theta_{2}$, upper record values of different sizes are generated from the MTR distribution.

2) Generate $p^{i}, \theta_{1}^{i}$ and $\theta_{2}^{i}, i=1,2, \cdots, \mu$, from the posterior PDF using MCMC algorithm.

3) Solving Equations (21), numerically, we get the 95\% MLPI for unobserved upper record values.

4) The MLPP for the future observation $t_{s}$, is computed using (22) when $p$ is known and (36) when $p$ and $\theta_{j}$ are unknown.

5) The 95\% BPI for unobserved upper record are obtained by solving Equations (29) and (30) when $p$ is known and (43) and (44) when $p$ and $\theta_{j}$ are unknown.

6) The BPP for the future observation $t_{s}$, is computed based on BSEL function using (31) when $p$ is known and (45) when $p$ and $\theta_{j}$ are unknown.

7) The BPP for the future observation $t_{s}$, is computed based on BLINX loss function using (33) when $p$ is known and (47) when $p$ and $\theta_{j}$ are unknown.

\subsection{Two Sample Prediction}

The following steps are used to obtain ML prediction (point and interval) and Bayesian prediction (point and interval) for future upper record value $s Y_{b}, b=1,2$.

1) For given values of $p, \theta_{1}$ and $\theta_{2}$, upper record values of different sizes are generated from the MTR distribution.

2) Generate $p^{i}, \theta_{1}^{i}$ and $\theta_{2}^{i}, i=1,2, \cdots, \mu$, from the posterior PDF using MCMC algorithm.

3) Solving equations (53) when $p$ is known and (66) when $p$ and $\theta_{j}$ are unknown we get the 95\% MLPI for unobserved upper record values.

4) The MLPP for the future observation $y_{b}$, is computed using (54) when $p$ is known and (67) when $p$ and $\theta_{j}$ are unknown.

5) The 95\% BPI for unobserved upper record are obtained by solving Equations (59) and (60) when $p$ is

Table 1. Point and 95\% interval predictors for the future upper record values $T_{s}^{*}$ when $\left(p=0.4, \theta_{1}=1.24915, \theta_{2}=3.19504\right.$, $\Omega=0.5$ ).

\begin{tabular}{|c|c|c|c|c|c|c|}
\hline \multirow{2}{*}{$\begin{array}{c}(r, s) \\
(3, r+1)\end{array}$} & \multicolumn{6}{|c|}{ Point predictions } \\
\hline & \multicolumn{3}{|c|}{ BLINEX $a=(0.01,2,3)$} & \multicolumn{2}{|c|}{ BSEL } & $\begin{array}{c}\mathrm{ML} \\
1.22518\end{array}$ \\
\hline$(3, r+2)$ & 1.50949 & 1.44461 & 1.42573 & & & 1.36665 \\
\hline$(5, r+1)$ & 1.54467 & 1.54467 & 1.50303 & & & 1.46006 \\
\hline$(5, r+2)$ & 1.73144 & 1.67009 & 1.65177 & & & 1.57457 \\
\hline$(7, r+1)$ & 1.4997 & 1.46631 & 1.4558 & & & 1.40629 \\
\hline$(7, r+2)$ & 1.68695 & 1.62164 & 1.60205 & & & 1.51416 \\
\hline \multirow{3}{*}{$(r, s)$} & \multicolumn{6}{|c|}{ Interval predictions } \\
\hline & \multicolumn{3}{|c|}{ Bayes } & \multicolumn{3}{|c|}{ ML } \\
\hline & $L$ & $U$ & length & $L$ & $U$ & length \\
\hline$(3, r+1)$ & 1.07653 & 2.16685 & 1.09032 & 1.07264 & 1.59494 & 0.522302 \\
\hline$(3, r+2)$ & 1.14454 & 2.61449 & 1.46995 & 1.03036 & 1.80551 & 0.77515 \\
\hline$(5, r+1)$ & 1.34499 & 2.34997 & 1.00498 & 1.34034 & 1.76244 & 0.422102 \\
\hline$(5, r+2)$ & 1.41102 & 2.76539 & 1.35437 & 1.30879 & 1.94403 & 0.635244 \\
\hline$(7, r+1)$ & 1.30032 & 2.31374 & 1.01342 & 1.29492 & 1.69241 & 0.397485 \\
\hline$(7, r+2)$ & 1.37071 & 2.71872 & 1.34801 & 1.2662 & 1.8674 & 0.601199 \\
\hline
\end{tabular}


Table 2. Point and $95 \%$ interval predictors for the future upper record values $T_{s}^{*}$ when $\left(p=0.391789, \theta_{1}=0.307317, \theta_{2}=\right.$ 3.33166, $\Omega=0.5)$.

\begin{tabular}{|c|c|c|c|c|c|c|}
\hline \multirow{3}{*}{$\begin{array}{c}(r, s) \\
(3, r+1)\end{array}$} & \multicolumn{6}{|c|}{ Point predictions } \\
\hline & \multicolumn{3}{|c|}{ BLINEX $a=(0.01,2,3)$} & \multicolumn{2}{|c|}{ BSEL } & \multirow{2}{*}{$\frac{\mathrm{ML}}{2.21747}$} \\
\hline & 2.322 & 2.23435 & 2.21354 & \multicolumn{2}{|c|}{2.32287} & \\
\hline$(3, r+2)$ & 2.68054 & 2.52154 & 2.48465 & \multicolumn{2}{|c|}{2.68226} & 2.4958 \\
\hline$(5, r+1)$ & 2.81243 & 2.74901 & 2.73223 & \multicolumn{2}{|c|}{2.813} & 2.74014 \\
\hline$(5, r+2)$ & 3.13233 & 3.01438 & 2.98352 & \multicolumn{2}{|c|}{3.13346} & 3.00112 \\
\hline$(7, r+1)$ & 2.66724 & 2.62719 & 2.61879 & \multicolumn{2}{|c|}{2.6678} & 2.62507 \\
\hline$(7, r+2)$ & 2.8745 & 2.79894 & 2.78385 & \multicolumn{2}{|c|}{2.87574} & 2.79966 \\
\hline & \multicolumn{6}{|c|}{ Interval predictions } \\
\hline \multirow[t]{2}{*}{$(r, s)$} & \multicolumn{3}{|c|}{ Bayes } & \multicolumn{3}{|c|}{ ML } \\
\hline & $L$ & $U$ & length & $L$ & $U$ & length \\
\hline$(3, r+1)$ & 1.91839 & 4.03255 & 2.11416 & 1.91487 & 2.94288 & 1.02801 \\
\hline$(3, r+2)$ & 1.80949 & 5.02409 & 3.2146 & 1.82977 & 3.35066 & 1.5209 \\
\hline$(5, r+1)$ & 2.46589 & 4.17173 & 1.70584 & 2.46339 & 3.42614 & 0.962751 \\
\hline$(5, r+2)$ & 2.54923 & 5.01563 & 2.46641 & 2.53061 & 3.82785 & 1.29724 \\
\hline$(7, r+1)$ & 2.44491 & 3.71097 & 1.26605 & 2.44436 & 3.08759 & 0.643231 \\
\hline$(7, r+2)$ & 2.39459 & 4.67043 & 2.27584 & 2.39746 & 3.37074 & 0.97328 \\
\hline
\end{tabular}

Table 3. Point and $95 \%$ interval predictors for the future upper record values $Y_{b}^{*}, b=1,2$ when $\left(p=0.4, \theta_{1}=1.24915, \theta_{2}=\right.$ 3.19504, $\Omega=0.5)$.

\begin{tabular}{|c|c|c|c|c|c|c|}
\hline \multirow{3}{*}{$\begin{array}{l}(r, b) \\
(3.1)\end{array}$} & \multicolumn{6}{|c|}{ Point predictions } \\
\hline & \multicolumn{4}{|c|}{ BLINEX $a=(0.01,2,3)$} & \multirow{2}{*}{$\begin{array}{c}\text { BSEL } \\
0.669789\end{array}$} & \multirow{2}{*}{$\frac{\text { ML }}{0.55108}$} \\
\hline & 0.669076 & \multicolumn{2}{|c|}{0.580084} & 0.552831 & & \\
\hline$(3.2)$ & 1.06708 & \multicolumn{2}{|c|}{0.940272} & 0.901294 & 1.06814 & 0.905675 \\
\hline (5.1) & 0.667379 & \multicolumn{2}{|c|}{0.581745} & 0.554524 & 0.668033 & 0.557414 \\
\hline$(5.2)$ & 1.05059 & \multicolumn{2}{|c|}{0.926826} & 0.888678 & 1.05157 & 0.879144 \\
\hline (7.1) & 0.637403 & \multicolumn{2}{|c|}{0.552511} & 0.526817 & 0.638057 & 0.506104 \\
\hline$(7.2)$ & 0.999761 & \multicolumn{2}{|c|}{0.874356} & 0.838862 & 1.00077 & 0.790802 \\
\hline & \multicolumn{6}{|c|}{ Interval predictions } \\
\hline \multirow[t]{2}{*}{$(r, b)$} & \multicolumn{3}{|c|}{ Bayes } & \multicolumn{3}{|c|}{ ML } \\
\hline & $L$ & $U$ & length $(C P)$ & $L$ & $U$ & length $(C P)$ \\
\hline (3.1) & 0.123947 & 2.03553 & $1.91158(96.16)$ & 0.0850494 & 1.47166 & $1.38661(97.70)$ \\
\hline$(3.2)$ & 0.37762 & 2.70191 & $2.32429(94.97)$ & 0.267226 & 1.9068 & $1.63957(98.54)$ \\
\hline (5.1) & 0.122991 & 1.97778 & $1.85479(95.90)$ & 0.0926795 & 1.36062 & $1.26794(96.50)$ \\
\hline$(5.2)$ & 0.377125 & 2.58594 & $2.20882(94.75)$ & 0.289275 & 1.75782 & $1.46854(97.63)$ \\
\hline (7.1) & 0.122419 & 1.93781 & $1.81539(96.01)$ & 0.0854495 & 1.20807 & $1.12263(95.03)$ \\
\hline$(7.2)$ & 0.376222 & 2.51787 & $2.14165(94.49)$ & 0.266284 & 1.55638 & $1.2901(95.43)$ \\
\hline
\end{tabular}


Table 4. Point and $95 \%$ interval predictors for the future upper record values $Y_{b}^{*}, b=1,2$ when $\left(p=0.391789, \theta_{1}=0.307317\right.$, $\left.\theta_{2}=3.33166, \Omega=0.5\right)$.

\begin{tabular}{cccccc}
\hline & \multicolumn{5}{c}{ Point predictions } \\
\cline { 2 - 5 }$(r, b)$ & \multicolumn{3}{c}{ BLINEX $a=(0.01,2,3)$} & BSEL & 0.827878 \\
\hline$(3.1)$ & 0.876869 & 0.696151 & 0.63902 & 0.878462 & 1.47625 \\
$(3.2)$ & 1.56559 & 1.22759 & 1.09298 & 1.56811 & 0.828237 \\
$(5.1)$ & 0.88791 & 0.707462 & 0.647328 & 0.889363 & 1.46954 \\
$(5.2)$ & 1.56375 & 1.25773 & 1.12298 & 1.56579 & 0.695196 \\
$(7.1)$ & 0.813281 & 0.645203 & 0.598027 & 0.814804 & 1.26946 \\
$(7.2)$ & 1.44473 & 1.15656 & 1.04976 & 1.44702 &
\end{tabular}

\begin{tabular}{ccccccc}
\hline & \multicolumn{9}{c}{ Interval predictions } \\
\cline { 2 - 7 }$(r, b)$ & \multicolumn{9}{c}{ Bayes } & & \multicolumn{2}{c}{ ML } & \\
\cline { 2 - 7 } & \multicolumn{1}{c}{ length $(C P)$} & $L$ & $U$ & length $(C P)$ \\
\hline$(3.1)$ & 0.100372 & 2.95917 & $2.8588(97.15)$ & 0.0974357 & 2.48911 & $2.39167(95.56)$ \\
$(3.2)$ & 0.320005 & 3.97264 & $3.65263(97.32)$ & 0.313335 & 3.18485 & $2.87151(95.36)$ \\
$(5.1)$ & 0.0981198 & 2.80057 & $2.70245(96.91)$ & 0.0915446 & 2.42924 & $2.33769(95.42)$ \\
$(5.2)$ & 0.319049 & 3.64706 & $3.32801(97.66)$ & 0.297965 & 3.08572 & $2.78776(95.16)$ \\
$(7.1)$ & 0.0971018 & 2.83087 & $2.73377(96.94)$ & 0.0826299 & 2.22201 & $2.13938(94.13)$ \\
$(7.2)$ & 0.304841 & 3.74134 & $3.4365(97.94)$ & 0.26343 & 2.87667 & $2.61324(93.33)$ \\
\hline
\end{tabular}

known and (72) and (73) when $p$ and $\theta_{j}$ are unknown.

6) The BPP for the future observation $y_{b}$, is computed based on BSEL function using (61) when $p$ is known and (74) when $p$ and $\theta_{j}$ are unknown.

7) The BPP for the future observation $y_{b}$, is computed based on BLINX loss function using (63) when $p$ is known and (76) when $p$ and $\theta_{j}$ are unknown.

8) Generate 10, 000 samples each of size $N=6$ from a MTR distribution, then calculate the coverage percentage (CP) of $Y_{b}$.

The computational (our) results were computed by using Mathematica 7.0. When $p$ is known, the prior parameters chosen as $v_{1}=2.3, v_{2}=2.7, \beta_{1}=0.5, \beta_{2}=1.3$ which yield the generated values of $\theta_{1}=1.24915$ and $\theta_{2}=3.19504$. While, in the case of four parameters are unknown the prior parameters $\left(b_{1}, b_{2}, c_{1}, c_{2}, d_{1}, d_{2}\right)$ chosen as $(1.2,2.3,2,2,0.3,3)$ which yield the generated values of $p=0.391789, \theta_{1}=0.307317, \theta_{2}=3.33166$. In Tables 1-4 point and 95\% interval predictors for the future upper record value are computed in case of the one- and two sample predictions, respectively.

\subsection{Conclusions}

It may be observed:
1) Point and 95\% interval predictors for future observations are obtained using a one-sample and two-sample schemes based on a MTR distribution. Our results are specialized to upper record values.

2) It is evident from all tables that, the lengths of the MLPI and BPI decrease as the sample size increase.

3) For fixed sample size $r$ the lengths of the MLPI and BPI increase by increasing $s$ or $b$.

4) The percentage coverage improves by use of a large number of observed values.

\section{REFERENCES}

[1] U. Kamps, "A Concept of Generalized Order Statistics," Journal of Statistical Planning and Inference, Vol. 48, No. 1, 1995, pp. 1-23.

doi:10.1016/0378-3758(94)00147-N

[2] M. Ahsanullah, "Generalized Order Statistics from Two Parameter Uniform Distribution," Communications in Statistics-Theory and Methods, Vol. 25, No. 10, 1996, pp. 2311-2318. doi:10.1080/03610929608831840

[3] M. Ahsanullah, "Generalized Order Statistics from Exponential Distributiuon," Journal of Statistical Planning and Inference, Vol. 85, No. 1-2, 2000, pp. 85-91. doi:10.1016/S0378-3758(99)00068-3

[4] U. Kamps and U. Gather, "Characteristic Property of 
Generalized Order Statistics for Exponential Distributions," Applicationes Mathematicae (Warsaw), Vol. 24, No. 4, 1997, pp. 383-391.

[5] E. Cramer and U. Kamps, "Relations for Expectations of Functions of Generalized Order Statistics," Journal of Statistical Planning and Inference, Vol. 89, No. 1-2, 2000, pp. 79-89. doi:10.1016/S0378-3758(00)00074-4

[6] M. Habibullah and M. Ahsanullah, "Estimation of Parameters of a Pareto Distribution by Generalized Order statistics," Communications in Statistics-Theory and Methods, Vol. 29, No. 7, 2000, pp. 1597-1609. doi: $10.1080 / 03610920008832567$

[7] Z. F. Jaheen, "On Bayesian Prediction of Generalized Order Statistics," Journal of Statistical Theory and Applications, Vol. 1, No. 3, 2002, pp.191-204.

[8] Z. F. Jaheen, "Estimation Based on Generalized Order Statistics from the Burr Model," Communications in Statistics-Theory and Methods, Vol. 34, No. 4, 2005, pp. 785-794. doi:10.1081/STA-200054408

[9] E. K. Al-Hussaini and A. A. Ahmad, "On Bayesian Predictive Distributions of Generalized Order Statistics," Metrika, Vol. 57, No. 2, 2003, pp. 165-176. doi:10.1007/s001840200207

[10] E. K. Al-Hussaini, "Generalized Order Statistics: Prospective and Applications," Journal of Applied Statistical Science, Vol. 13, No. 1, 2004, pp. 59-85.

[11] A. A. Ahmad and T. A. Abu-Shal, "Recurrence Relations for Moment Generating Functions of Nonadjacent Generalized Order Statistics Based on a Class of Doubly Truncated Distributions," Journal of Statistical Theory and Applications, Vol. 6, No. 2, 2007, pp. 174-189.

[12] A. A. Ahmad and T. A. Abu-Shal, "Recurrence Relations for Moment Generating Functions of Generalized Order Statistics from Doubly Truncated Continuous Distributions," Journal of Statistical Theory and Applications, Vol. 6, No. 2, 2008, pp. 243-257.

[13] A. A. Ahmad, "Relations for Single and Product Moments of Generalized Order Statistics from Doubly Truncated Burr type XII Distribution," Journal of the Egyptian Mathematical Society, Vol. 15, No. 1, 2007, pp. 117128.

[14] A. A. Ahmad, "Single and Product Moments of Generalized Order Statistics from Linear Exponential Distribution," Communications in Statistics-Theory and Methods, Vol. 37, No. 8, 2008, pp. 1162-1172. doi:10.1080/03610920701713344

[15] Z. A. Aboeleneen, "Inference for Weibull Distribution under Generalized Order Statistics," Mathematics and Computers in Simulation, Vol. 81, No. 1, 2010, pp. 26-36. doi:10.1016/j.matcom.2010.06.013

[16] S. Abu El Fotouh, "Estimation for the Parameters of the Weibull Extension Model Based on Generalized Order Statistics," International Journal of Contemporary Mathematical Sciencess, Vol. 6, No. 36, 2011, pp. 1749-1760.

[17] S. F. Ateya, "Prediction under Generalized Exponential Distribution Using MCMC Algorithm," International Mathematical Forum, Vol. 6, No. 63, 2011, pp. 3111-3119.
[18] S. F. Ateya and A. A. Ahmad, "Inferences Based on Generalized Order Statistics under Truncated Type I Generalized Logistic Distribution," Statistics, Vol. 45, No. 4, 2011, pp. 389-402. doi:10.1080/02331881003650149

[19] B. S. Everitt and D. J. Hand, "Finite Mixture Distributions," Cambridge University Press, Cambridge, 1981.

[20] D. M. Titterington, A. F. M. Smith and U. E. Makov, "Statistical Analysis of Finite Mixture Distributions," John Wiley and Sons, New York, 1985.

[21] K. E. Ahmad, "Identifiability of Finite Mixtures Using a New Transform," Annals of the Institute of Statistical Mathematics, Vol. 40, No. 2, 1988, pp. 261-265. doi:10.1007/BF00052342

[22] G. J. McLachlan and K. E. Basford, "Mixture Models: Inferences and Applications to Clustering," Marcel Dekker, New York, 1988.

[23] Z. F. Jaheen, "On Record Statistics from a Mixture of Two Exponential Distributions," Journal of Statistical Computation and Simulation, Vol. 75, No. 1, 2005, pp. 111. doi:10.1080/00949650410001646924

[24] K. E. Ahmad, Z. F. Jaheen and Heba S. Mohammed, "Bayesian Prediction Based on Type-I Censored Data from a Mixture of Burr Type XII Distribution and Its Reciprocal," Statistics, Vol. 1, No. 1, 2011, pp. 1-11. doi:10.1080/02331888.2011.555550

[25] E. K. AL-Hussaini and M. Hussein, "Estimation under a Finite Mixture of Exponentiated Exponential Components Model and Balanced Square Error Loss," Open Journal of Statistics, Vol. 2, No. 1, 2012, pp. 28-38. doi:10.4236/ojs.2012.21004

[26] M. A. M. Ali-Mousa, "Bayesian Prediction Based on Pareto Doubly Censored Data," Statistics, Vol. 37, No. 1, 2003, pp. 65-72. doi:10.1080/0233188021000004639

[27] A. S. Papadapoulos and W. J. Padgett, "On Bayes Estimation for Mixtures of Two Exponential-Life-Distributions from Right-Censored Samples," IEEE Transactions on Reliability, Vol. 35, No. 1, 1986, pp. 102-105. doi:10.1109/TR.1986.4335364

[28] A. F. Attia, "On Estimation for Mixtures of 2 Rayleigh Distribution with Censoring," Microelectronics Reliability, Vol. 33, No. 6, 1993, pp. 859-867. doi:10.1016/0026-2714(93)90259-2

[29] K. E. Ahmad, H. M. Moustafa and A. M. Abd-El-Rahman, "Approximate Bayes Estimation for Mixtures of Two Weibull Distributions under Type II Censoring," Journal of Statistical Computation and Simulation, Vol. 58, No. 3, 1997, pp. 269-285. doi:10.1080/00949659708811835

[30] A. A. Soliman, "Estimators for the Finite Mixture of Rayleigh Model Based on Progressively Censored Data," Communications in Statistics-Theory and Methods, Vol. 35, No. 5, 2006, pp. 803-820. doi:10.1080/03610920500501379

[31] M. Saleem and M. Aslam, "Bayesian Analysis of the Two Component Mixture of the Rayleigh Distribution Assuming the Uniform and the Jeffreys Priors," Journal of Applied Statistical Science, Vol. 16, No. 4, 2008, pp. 105-113. 
[32] M. Saleem and M. Aslam, "On Prior Selection for the Mixture of Rayleigh Distribution Using Predictive Intervals," Pakistan Journal of Statistics, Vol. 24, No. 1, 2007, pp. 21-35.

[33] M. Saleem and M. Irfan, "On Properties of the Bayes estimates of the Rayleigh Mixture Parameters: A Simulation Study," Pakistan Journal of Statistics, Vol. 26, No. 3, 2010, pp. 547-555.

[34] A. Zellner, "Bayesian and Non-Bayesian Estimation Using Balanced Loss Functions," In: J. O. Berger and S. S. Gupta, Eds., Statistical Decision Theory and Methods. V, Springer, New York, 1994, pp. 339-390.

[35] M. J. Jozani, E. Marchand and A. Parsian, "Bayes Estimation under a General Class of Balanced Loss Functions," Universite de Sherbrooke, Sherbrooke, 2006.
[36] E. K. Al-Hussaini and K. E. Ahmad, "On the Identifiability of Finite Mixtures of Distributions," IEEE Transactions on Information Theory, Vol. 27, No. 5, 1981, pp. 664-668. doi:10.1109/TIT.1981.1056389

[37] K. E. Ahmad and E. K. Al-Hussaini, "Remarks on the Non-Identifiability of Mixtures of Distributions," Annals of the Institute of Statistical Mathematics, Vol. 34, No. 1, 1982, pp. 543-544. doi:10.1007/BF02481052

[38] S. J. Press, "Subjective and Objective Bayesian Statistics: Principles, Models and Applications," Wiley, New York, 2003.

[39] J. Aitchison and I.R. Dunsmore, "Statistical Prediction Analysis," Cambridge University Press, Cambridge, 1975. 